\title{
Extended-time of Noninvasive Positive Pressure Ventilation Improves Tissue Perfusion after Coronary Artery Bypass Surgery: a Randomized Clinical Trial
}

\author{
Mara L. S. Nasrala1,2, PT, MSc; Douglas W. Bolzan', PT, PhD; Yumi G. Lage2,3, PT; Fabiana S. Prado 2,3, PT; Ross Arena4, PT, \\ PhD; Paulo R. L. Lima ${ }^{2,3}$, MD; Gibran Feguri ${ }^{2,3}$, MD, MSc; Ageo M. C. Silva ${ }^{5}$, MD, PhD; Natasha O. Marcondi ${ }^{1}$, PT, MSc; \\ Nelson Hossne ${ }^{1}$, MD, PhD; Solange Guizilini ${ }^{1,6}, \mathrm{PT}, \mathrm{PhD}$; Walter J. Gomes ${ }^{1}$, PhD
}

DOI: $10.21470 / 1678-9741-2017-0232$

\begin{abstract}
Objective: To compare the effects of extended- versus shorttime noninvasive positive pressure ventilation on pulmonary function, tissue perfusion, and clinical outcomes in the early postoperative period following coronary artery bypass surgery in patients with preserved left ventricular function.

Methods: Patients were randomized into two groups according to noninvasive positive pressure ventilation intensity: short-time noninvasive positive pressure ventilation $n=20$ (S-NPPV) and extended-time noninvasive positive pressure ventilation $n=21$ (E-NPPV). S-NPPV was applied for 60 minutes during immediate postoperative period and 10 minutes, twice daily, from postoperative days 1-5. E-NPPV was performed for at least six hours during immediate postoperative period and 60 minutes, twice daily, from postoperative days 1-5. As a primary outcome, tissue perfusion was determined by central venous oxygen saturation and blood lactate level measured after anesthetic induction, immediately after extubation and following noninvasive positive pressure ventilation protocols. As
\end{abstract}

a secondary outcome, pulmonary function tests were performed preoperatively and in the postoperative days 1, 3, and 5; clinical outcomes were recorded.

Results: Significant drop in blood lactate levels and an improvement in central venous oxygen saturation values in the E-NPPV group were observed when compared with S-NPPV group after study protocol $(P<0.01)$. The E-NPPV group presented higher preservation of postoperative pulmonary function as well as lower incidence of respiratory events and shorter postoperative hospital stay $(P<0.05)$.

Conclusion: Prophylactic E-NPPV administered in the early postoperative period of coronary artery bypass surgery resulted in greater improvements in tissue perfusion, pulmonary function and clinical outcomes than S-NPPV, in patients with preserved left ventricular function.

Trial Registration: Brazilian Registry of Clinical trial RBR7sqj78 - http://www.ensaiosclinicos.gov.br

Keywords: Coronary Artery Bypass. Lactic Acid/Blood. Lung/ Physiology. Forced Expiratory Volume. Positive-Pressure Respiration.

\begin{tabular}{|c|c|c|c|}
\hline \multicolumn{4}{|c|}{ Abbreviations, acronyms \& symbols } \\
\hline BIPAP & $=$ Bilevel positive airway pressure & IPO & $=$ Immediate postoperative period \\
\hline BMI & $=$ Body mass index & LITA & $=$ Left internal thoracic artery \\
\hline CABG & $=$ Coronary artery bypass surgery & LVEF & $=$ Left ventricular ejection fraction \\
\hline CPB & $=$ Cardiopulmonary bypass & NPPV & $=$ Noninvasive positive pressure ventilation \\
\hline CVP & $=$ Central venous pressure & PEEP & $=$ Positive end-expiratory pressure \\
\hline E-NPPV & = Extended-time noninvasive positive pressure ventilation & POD & $=$ Postoperative day \\
\hline FEV $_{1}$ & $=$ Forced expiratory volume in 1 second & $\mathrm{ScvO}_{2}$ & $=$ Central venous oxygen saturation \\
\hline $\mathrm{FiO}_{2}$ & $=$ Inspired oxygen fraction & S-NPPV & $=$ Short-time noninvasive positive pressure ventilation \\
\hline FVC & = Forced vital capacity & $\mathrm{SpO}_{2}$ & $=$ Arterial oxygen saturation \\
\hline ICU & $=$ Intensive care unit & & \\
\hline
\end{tabular}

'Cardiology and Cardiovascular Surgery Disciplines, Escola Paulista de Medicina, Universidade Federal de São Paulo (EPM-UNIFESP), São Paulo, SP, Brazil.

${ }^{2}$ Physical Therapy Department, Hospital Santa Rosa, Cuiabá, MT, Brazil.

3Physical Therapy Department, Hospital São Mateus, Cuiabá, MT, Brazil.

${ }^{4}$ Department of Physical Therapy, College of Applied Health Sciences, University of Illinois Chicago, Chicago, IL, USA.

5Public Health Department, Universidade Federal do Mato Grosso (UFMT), Cuiabá, MT, Brazil.

${ }^{6}$ Department of the Human Movement Sciences, Universidade Federal de São Paulo (UNIFESP), São Paulo, SP, Brazil.

This study was carried out at Universidade Federal de Mato Grosso (UFMT), Cuiabá, MT, Brazil.
Financial support: Fundação de Amparo à Pesquisa do Estado de Mato Grosso. No conflict of interest.

Correspondence Address:

Solange Guizilini

Department of Human Movement Sciences - Universidade Federal de São Paulo Rua Silva Jardim, 136, Edifício Central - Santos, SP, Brasil -

Zip code: 11015-020

E-mail: s_guizilini@yahoo.com.br 


\section{INTRODUCTION}

Elevated blood lactate level and low central venous oxygen saturation $\left(\mathrm{ScVO}_{2}\right)$ have been independently associated with an increased risk of complications and longer postoperative hospital stay following cardiac surgery, contributing to increased morbidity and mortality after coronary artery bypass surgery $(C A B G)^{[1-3]}$. In addition, several studies have shown that postoperative pulmonary dysfunction following CABG is inevitable, which could increase the occurrence of respiratory complications and delay recovery ${ }^{[4-6]}$.

Noninvasive positive pressure ventilation (NPPV) has been used to accelerate the recovery of pulmonary function as well as to prevent and treat postoperative pulmonary complications. Previous evidence indicates a significant drop in blood lactate concentration 60 minutes following NPPV ${ }^{[7]}$.

To date, no study has addressed the influence of early use of NPPV on key measures and clinical outcomes following cardiac surgery. In this context, the aim of the current study was to compare the effects of extended-versus short-time prophylactic NPPV, applied in the early postoperative period following $C A B G$, on pulmonary function parameters, tissue perfusion determined by $\mathrm{ScvO}_{2}$, blood lactate level, and clinical outcomes. We hypothesized that NPPV would provide numerous beneficial effects and that the extended-time mode would be superior to short-time NPPV.

\section{METHODS}

This randomized controlled trial was conducted between June 2013 and May 2014, at Santa Rosa and São Mateus Hospitals, Brazil. All ethical aspects were respected, with approval of the institutions' Clinical Ethical Research Committees. All subjects were informed about the study and they have signed a written consent form prior to the enrollment.

\section{Patients}

Patients undergoing elective first-time on-pump CABG were prospectively included in the current study. Inclusion criteria were: both genders and 18 years of age or older. Inability to perform spirometry, hemodynamic instability, left ventricular ejection fraction less than 45\%, emergency surgery, chronic or acute pulmonary disease, intraoperative death, renal failure (creatinine $>1.3 \mathrm{mg} / \mathrm{dL}$ ), anatomical abnormalities interfering with NPPV mask fit, obesity [i.e., body mass index (BMI) > 30], steroid treatment, and uncooperative state served as primary exclusion criteria.

The patients were prospectively randomized into two groups: short-time NPPV (S-NPPV; $\mathrm{n}=20$ ) and extended-time NPPV (E-NPPV; $n=21$ ). A random sequence was performed through a software on "random.org" and allocation secrecy was kept by numbered, sealed, opaque envelopes.

\section{Surgical Procedure}

All patients received the same anesthetic regimen during CABG. Anesthesia was induced in a routine manner with etomidate and midazolam and maintained with fentanyl and sevoflurane ( $0.5 \%$ to $1 \%)$. Mechanical ventilation was started with volume-controlled ventilation at the following settings: 1) tidal volume at $8 \mathrm{ml} / \mathrm{kg}$ of predicted body weight; 2) positive endexpiratory pressure (PEEP) at $0 \mathrm{cmH}_{2} \mathrm{O} ; 3$ ) inspiration/expiration ratio at $1: 2 ; 4)$ inspired oxygen fraction $\left(\mathrm{FiO}_{2}\right)$ set to keep oxygen saturation above $90 \%$; and 5) respiratory rate adjusted to achieve a $\mathrm{PaCO}_{2}$ between 35 and $45 \mathrm{mmHg}$. During the operation, mean arterial pressure, central venous pressure (CVP), arterial blood gas, temperature, urine output, electrocardiography, and heart rate were continuously monitored.

Operation was performed through a median sternotomy, using the left internal thoracic artery (LITA) graft, which was harvested according to the skeletonized technique and complemented with additional saphenous vein grafts. Meticulous care was routinely taken to preserve the pleura integrity during LITA harvesting. In all patients, before chest closure, in the presence of incidental left pleura opening, a soft tubular PVC drain was inserted and exteriorized at the subxiphoid region and positioned in the left costophrenic sinus. In all subjects, a mediastinal drain was also placed via a subxiphoid entry.

Cardiopulmonary bypass (CPB) was established with ascending aorta cannulation and single cannula venous drainage, after systemic heparinization to keep the activated coagulation time above 480 seconds. Myocardial protection was achieved using intermittent hypothermic antegrade blood cardioplegia, associated with systemic mild hypothermia $\left(34^{\circ} \mathrm{C}\right)$.

\section{Postoperative Management}

All patients were transferred to the intensive care unit (ICU) and ventilated on volume-controlled ventilation using the following parameters: 12-14 breaths/minute with a $\mathrm{FiO}_{2}$ level set to maintain arterial oxygen saturation $\left(\mathrm{SpO}_{2}\right)$ above $90 \%$; inspiratory/expiratory ratio of 1:2; PEEP of $5 \mathrm{cmH}_{2} \mathrm{O}$; and pressure support to maintain a tidal volume of $8 \mathrm{ml} / \mathrm{kg}$ of predicted body weight. Extubation was performed when patients were hemodynamically stable and alert to maintain self-ventilation and good blood gas values. All patients received the same analgesic protocol (100 mg of tramadol chlorhydrate, 4 times a day) administered until postoperative day (POD) 5. Patients also underwent daily physical therapy sessions until discharge. Chest tubes were routinely removed on POD2 and patients had daily chest X-ray.

\section{Study Design}

Following extubation, all E-NPPV patients received NPPV with bilevel positive airway pressure (BIPAP) for at least six hours in the immediate postoperative period (IPO) and 60 minutes, twice a day, from POD1 to POD5. S-NPPV patients received NPPV with BIPAP administered for 1 hour in the IPO period and 10 minutes, twice a day, from POD1 to POD5, according to the ICU routine. In both groups, during NPPV application, patients were in semirecumbent position, with the head of the bed elevated at $45^{\circ}$. BiPAP ${ }^{\otimes}$ Synchrony ${ }^{\oplus}$ equipment (Respironics) was used with an adjustable face mask with the following parameters: inspiratory positive airway pressure sufficient to ensure a tidal volume of 8 $\mathrm{ml} / \mathrm{kg}$; and PEEP of $10 \mathrm{cmH}_{2} \mathrm{O}$ with $\mathrm{FiO}_{2}$ adjusted to maintain $\mathrm{SpO}_{2}>90 \%$. 


\section{End Points}

As a primary outcome, tissue perfusion was determined by $\mathrm{ScvO}_{2}$ and blood lactate level. As a secondary outcome, pulmonary function and clinical outcomes were assessed.

\section{Tissue Perfusion}

For tissue perfusion analysis, blood samples directly drawn from the CVP catheter were analyzed to assess the $\mathrm{ScvO}_{2}$ and, simultaneously, blood samples collected from the arterial catheter were used to evaluate blood lactate levels. Low $\mathrm{ScvO}_{2}$ was defined as $<65 \%$ and hyperlactatemia was defined as a blood lactate level $>3 \mathrm{mmol} /$. Blood samples were collected in three moments at the IPO: intraoperative period (after anesthesia induction and invasive mechanical ventilation), immediately after extubation (spontaneous ventilation), and immediately after the NPPV protocol.

\section{Pulmonary Function}

Forced vital capacity (FVC) and forced expiratory volume in 1 second $\left(F E V_{1}\right)$ were evaluated at the bedside on the day before the operation and repeated on POD1, 3, and 5 (after NPPV protocols) by the same respiratory physiotherapist, using a portable spirometer (Spirobank G, MIR, Rome, Italy), according to the American Thoracic Society standards ${ }^{[8]}$.

\section{Clinical Outcomes}

Length of mechanical ventilation and duration of postoperative hospital stay were recorded for all patients. A radiologist who was blinded to subject group allocation evaluated chest roentgenograms taken preoperatively and through POD1 to POD5. Respiratory events were also evaluated (atelectasis, pleural effusions, and pneumonia). Pleural effusion was considered relevant when exceeding the phreno-costal angle and fluid drainage was monitored hourly ${ }^{[4]}$. Atelectasis was acknowledged when a clear atelectasis radiological shadow exceeded $15 \mathrm{~mm}$ in width ${ }^{[4]}$; linear atelectasis was disregarded in this study. Pneumonia was defined by the presence on chest radiographs with new or persistent pulmonary infiltrates not otherwise explained, in combination with at least two of the following criteria: body temperature of $>38^{\circ} \mathrm{C}$; leukocytosis $\left(>10,000\right.$ cells $\left./ \mathrm{mm}^{3}\right)$; and purulent respiratory secretions ${ }^{[9]}$. Assessors blinded to group allocation documented the incidence of respiratory events.

\section{Statistical Analysis}

Data are reported as mean \pm standard deviation. Based on previous studies ${ }^{[4]}$, sample size calculation was based on FVC at POD1, considering a significance level of 5\% and $80 \%$ power to detect a difference between groups of at least a $400 \mathrm{ml}$ decrease compared to the preoperative period. This assumption suggested a sample of 40 patients, resulting in a total of 60 patients recruited to account for patients not completing the study ${ }^{[10]}$. Initially, the Kolgomorov-Smirnov test was applied to determine the distribution of variables. When variables were compared between groups, we used the unpaired Student's t-test; and Mann-Whitney test was used when deemed necessary. For intragroup analysis, we used the paired Student's t-test; and ANOVA was used for repeated measures, as appropriate. For categorical data, the Pearson's chi-square test was performed. The Pearson correlation coefficient was used to evaluate associations.

In this study, clinically relevant threshold values of $3 \mathrm{mmol} / \mathrm{l}$ for blood lactate level and $65 \%$ for the $\mathrm{ScvO}_{2}$ were used. Trend analysis of these variables was performed using simple linear regression models. The construction of scatter plots of the variables showed in all cases that a linear evaluation could be assumed, which supported the use of this model. Simple linear regression models were adjusted for each group. As a measure of precision of these models, we used the coefficient of determination $\left(r^{2}\right)$, later transformed into Pearson's correlation coefficient. Statistical analysis was performed by computerized statistical program (SPSS13.0, Chicago, IL, USA) software. For all statistical tests, a $P$-value $<0.05$ defined statistical significance.

\section{RESULTS}

During the study period, 70 patients were assessed for eligibility. From that sample, 16 were excluded, 54 were randomized, and 41 were, in fact, analyzed (Figure 1).

The groups were homogeneous; pre- and intraoperative patients' characteristics are summarized in Table 1. Following extubation, patients in the E-NPPV group received prophylactic NPPV for $6.21 \pm 0.44$ hours.

A significant increase in blood lactate levels and a drop of $\mathrm{SCVO}_{2}$ were observed in both groups after extubation in comparison with pre-anesthetic values $(P<0.05)$. A strong negative correlation ( $r=-0.84)$ was observed between blood lactate levels and $\mathrm{ScvO}_{2}$ after trend analysis by linear regression, with all patients breathing spontaneously (before NPPV application) during the IPO period $(P<0.001)$.

After NPPV, a significant drop in blood lactate levels and an improvement in $\mathrm{SCvO}_{2}$ values were observed in the E-NPPV group when compared with S-NPPV group $(P<0.05)$ (Figures 2 and 3 , respectively).

In patients with blood lactate levels above $3 \mathrm{mmol} / \mathrm{l}$ (considering baseline values as 100\%) during the IPO period, the use of E-NPPV lead to a 30.3\% reduction in blood lactate levels. Conversely, the S-NPPV group demonstrated a 15.6\% decline in blood lactate levels during the IPO period. In patients with $\mathrm{ScvO}_{2}$ values below 65\% during the IPO period (considering baseline values as 100\%), E-NPPV was able to increase in $23.3 \%$ the $\mathrm{ScvO}_{2}$ values, while in the S-NPPV group a 1.2\% further decline was seen.

In Figure 4, the distribution of both groups was graphically analyzed regarding to blood lactate levels and $\mathrm{ScvO}_{2}$ values. Nine (45\%) S-NPPV patients and two (9.5\%) E-NPPV patients had blood lactate peak above $3 \mathrm{mmol} / \mathrm{I}$ and $\mathrm{ScvO}_{2}<65 \%$ (upper left quadrant). In the lower right quadrant, 17 (80.9\%) E-NPPV patients and eight (40\%) S-NPPV patients showed peak blood lactate levels below $3 \mathrm{mmol} / \mathrm{l}$ and $\mathrm{ScvO}_{2}>65 \%$.

A significant impairment in FVC and $\mathrm{FEV}_{1}$ until POD5 was 


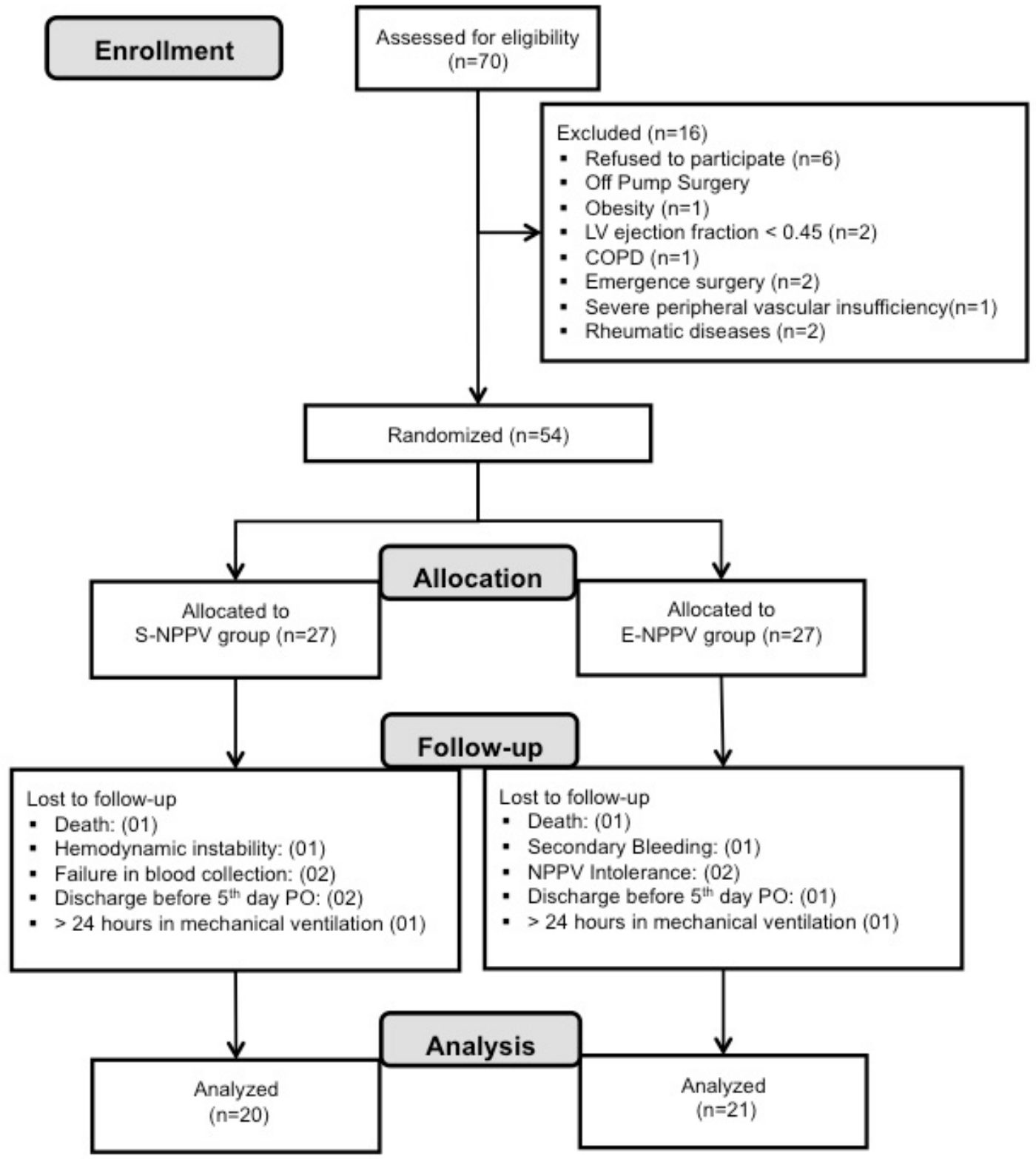

Fig. 1 - Flowchart of consecutive CABG patients enrolled in the study.

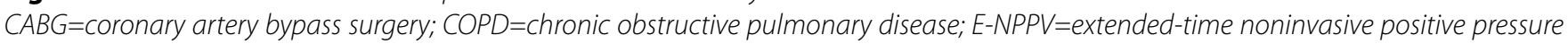
ventilation; $L V=$ left ventricular; $P O=$ postoperative; $S-N P P V=$ short-time noninvasive positive pressure ventilation

observed in both groups in comparison with the preoperative data $(P<0.01)$. However, the E-NPPV group presented with higher FVC and FEV 1 values on POD1, 3 and 5 than the S-NPPV group. Significant differences were found in $\mathrm{FEV}_{1}$ on POD1 between these groups. FVC and $\mathrm{FEV}_{1}$ were significantly different between S-NPPV and E-NPPV groups on POD5 (Table 2).

Hospital stay after CABG was significantly shorter in the E-NPPV group than in the S-NPPV group $(P<0.05)$. Moreover, the incidence of respiratory events on POD5 was greater in the S-NPPV group than in the E-NPPV group (Table 3).

\section{DISCUSSION}

Prophylactic E-NPPV intervention demonstrated a positive impact on tissue perfusion, preservation of pulmonary function, and clinical outcomes compared to S-NPPV in the early postoperative period after CABG in this group of patients with preserved cardiac function. To our knowledge, this is the first study comparing the aforementioned outcomes between E-NPPV and S-NPPV after a major cardiac surgery.

A previous investigation [11] demonstrated that the prophylactic use of E-NPPV is able to promote a better 
Table 1. Pre- and intraoperative patients' characteristics.

\begin{tabular}{|c|c|c|c|}
\hline Variables & $\begin{array}{c}\text { S-NPPV Group } \\
(n=20)\end{array}$ & $\begin{array}{l}\text { E-NPPV Group } \\
(n=21)\end{array}$ & $P$ value \\
\hline Age (years) & $59.7 \pm 11.4$ & $58.6 \pm 7.3$ & 0.36 \\
\hline Men \% (n) & $85.0(17)$ & $76.2(16)$ & 0.17 \\
\hline $\mathrm{BMI}\left(\mathrm{kg} / \mathrm{m}^{2}\right)$ & $27.4 \pm 5.0$ & $27.7 \pm 4.0$ & 0.80 \\
\hline LVEF (\%) & $61.2 \pm 14.6$ & $63.0 \pm 11.3$ & 0.66 \\
\hline CPB time (min) & $71.1 \pm 19.9$ & $70.5 \pm 27.0$ & 0.71 \\
\hline Aortic cross-clamp time (min) & $55.2 \pm 15.2$ & $53.2 \pm 20.5$ & 0.52 \\
\hline Operative time (h) & $4.2 \pm 1.3$ & $3.7 \pm 0.9$ & 0.51 \\
\hline Grafts per patient (n) & $2.9 \pm 1.0$ & $2.6 \pm 0.9$ & 0.26 \\
\hline \multicolumn{4}{|l|}{ Pulmonary function } \\
\hline FVC (I) & $3.3 \pm 0.9$ & $3.1 \pm 0.8$ & 0.40 \\
\hline$\%$ predicted & $94.21 \pm 18.2$ & $91.72 \pm 15.9$ & 0.35 \\
\hline $\mathrm{FEV}_{1}(\mathrm{I})$ & $2.8 \pm 0.7$ & $2.9 \pm 0.7$ & 0.42 \\
\hline$\%$ predicted & $88.7 \pm 18.0$ & $90.1 \pm 16.1$ & 0.29 \\
\hline Pleurotomy \% (n) & $15.0(3)$ & $9.5(2)$ & 0.30 \\
\hline
\end{tabular}

Data are shown as mean \pm standard deviation.

$\mathrm{BMI}=$ body mass index; $\mathrm{CPB}=$ cardiopulmonary bypass; $\mathrm{E}-\mathrm{NPPV}=$ extended-time noninvasive positive pressure ventilation; $\mathrm{FEV}_{1}=\mathrm{forced}$ expiratory volume in 1 second; FVC=forced vital capacity; LVEF=left ventricular ejection fraction; S-NPPV=short-time noninvasive positive pressure ventilation

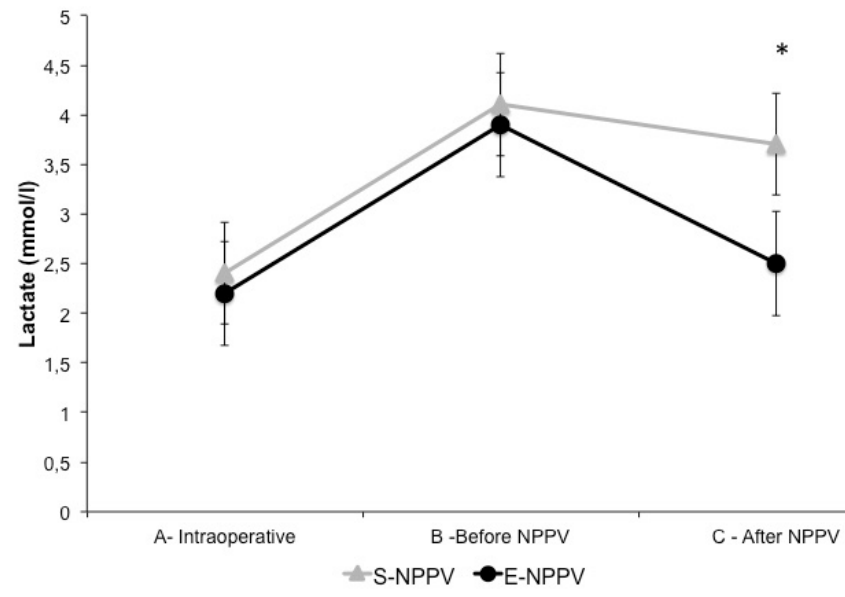

Fig. 2 - Sequential changes of blood lactate level before and after NPPV.

${ }^{*} P<0.05$.

$A=$ intraoperative (after anesthesia induction and invasive mechanical ventilation); $B=$ before NPPV (spontaneous ventilation); $C=$ after NPPV (immediately after NPPV protocol); E-NPPV=extendedtime noninvasive positive pressure ventilation; S-NPPV=short-time noninvasive positive pressure ventilation

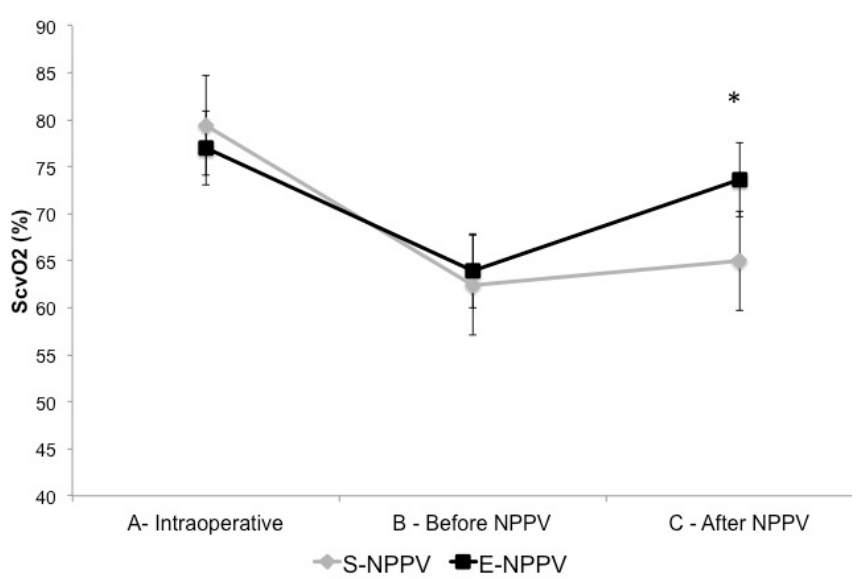

Fig. 3 - Sequential changes of $\mathrm{SCVO}_{2}$ before and after NPPV. ${ }^{*} P<0.05$.

$A=$ intraoperative (after anesthesia induction and invasive mechanical ventilation); $B=$ before NPPV (spontaneous ventilation); $C=$ after NPPV (immediately after NPPV protocol); $E-N P P V=$ extendedtime noninvasive positive pressure ventilation; S-NPPV=short-time noninvasive positive pressure ventilation; $\mathrm{SCvO}_{2}=$ central venous oxygen saturation 


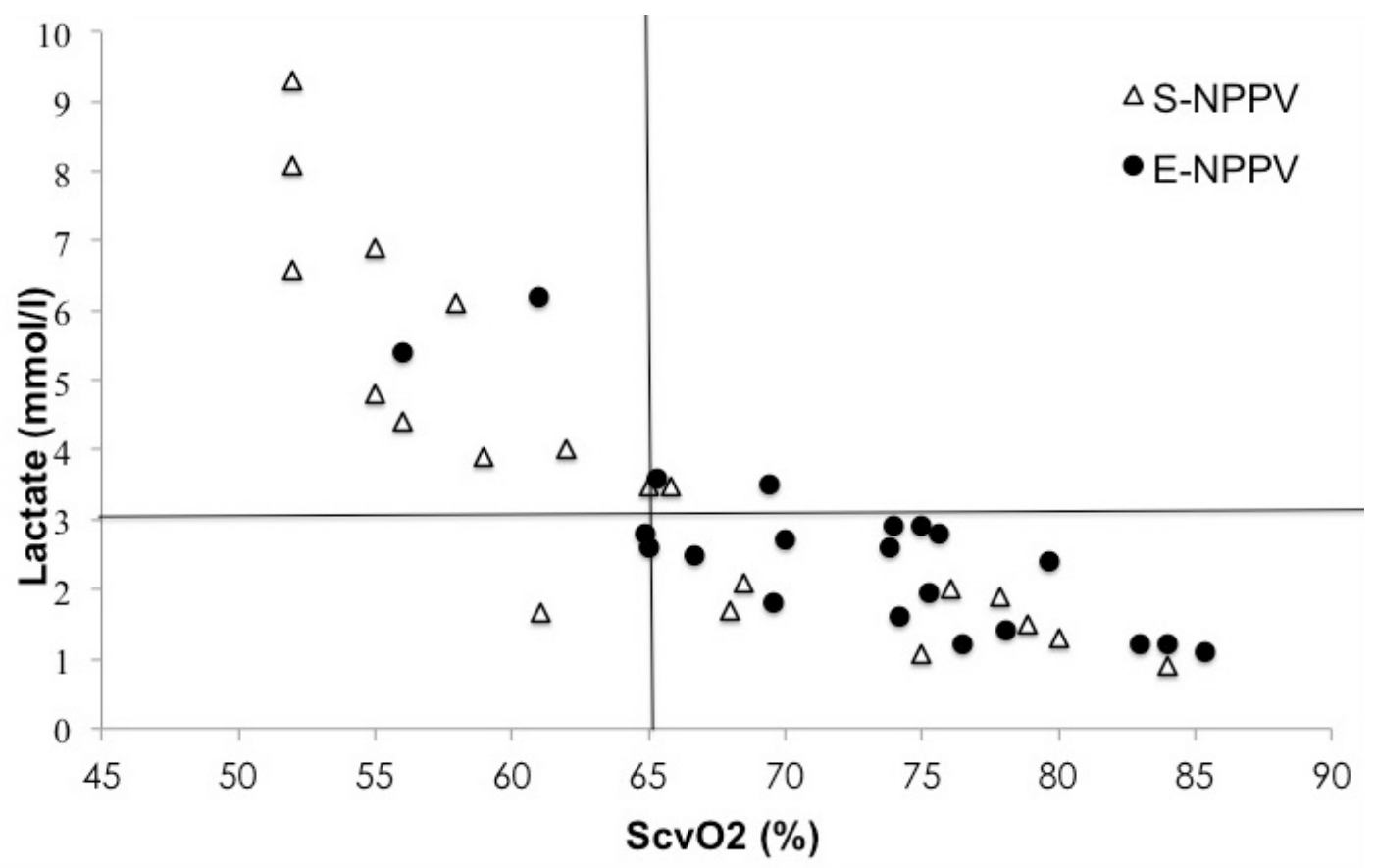

Fig. 4 - Patients' distribution according to cut-off values of 65\% $\left(\mathrm{ScVO}_{2}\right)$ and $3 \mathrm{mmol} / \mathrm{L}$ (lactate) after NPPV protocol. E-NPPV=extended-time noninvasive positive pressure ventilation; S-NPPV=short-time noninvasive positive pressure ventilation; $S \mathrm{Cv} \mathrm{O}_{2}=\mathrm{central}$ venous oxygen saturation

Table 2. Pulmonary function test values on postoperative days (POD) 1,3 , and 5 , in percentage of preoperative values.

\begin{tabular}{|c|c|c|c|c|}
\hline \multirow[t]{2}{*}{ Variables } & \multicolumn{2}{|c|}{$\begin{array}{c}\text { S-NPPV Group } \\
(n=20)\end{array}$} & \multicolumn{2}{|c|}{$\begin{array}{c}\text { E-NPPV Group } \\
(n=21)\end{array}$} \\
\hline & FVC (\%) & $\mathrm{FEV}_{1}(\%)$ & FVC (\%) & $\mathrm{FEV}_{1}(\%)$ \\
\hline POD1 & $43.34 \pm 13.8$ & $45.32 \pm 15.7$ & $54.34 \pm 24.5^{*}$ & $55.84 \pm 12.4^{*}$ \\
\hline POD3 & $47.34 \pm 19.9$ & $49.91 \pm 17.5$ & $65.56 \pm 17.4^{*}$ & $72.38 \pm 15.1^{*}$ \\
\hline POD5 & $67.75 \pm 16.3$ & $58.04 \pm 12.0$ & $79.87 \pm 18.5^{*}$ & $80.45 \pm 16.3^{*}$ \\
\hline
\end{tabular}

Data are shown as mean \pm standard deviation. FVC and $\mathrm{FEV}_{1}$ are expressed in percentage considering $100 \%$ preoperative baseline value. ${ }^{*} P<0.05$ for comparison between groups.

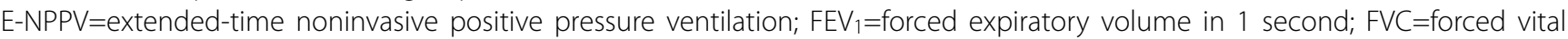
capacity; S-NPPV=short-time noninvasive positive pressure ventilation

improvement in arterial oxygenation and some clinical outcomes than the S-NPPV. However, the above-mentioned study did not evaluate the effects of NPPV on tissue perfusion. Another study ${ }^{[7]}$ suggested that NPPV could be considered an effective and safe therapy to minimize dyspnea, improve tissue perfusion, and decrease arrhythmia frequency, reintubation rate, length of ICU stay, and mortality in patients after cardiac surgery when compared to oxygen therapy alone. Therefore, the novel aspects of our study were the influence of E-NPPV and S-NPPV application on tissue perfusion, pulmonary function, and clinical outcomes in patients who underwent CABG.

Recently, an investigation documented that a significant impairment in tissue perfusion (i.e. elevated lactate level and low $\mathrm{ScvO}_{2}$ ) could delay operation recovery, increase the risk of complications, and lead to a longer postoperative hospital stay, contributing to the risk of morbidity and mortality following $\mathrm{CABG}^{[1-3]}$. As a result, special interest has been given to strategies to improve tissue perfusion ${ }^{[12]}$.

In agreement with other studies ${ }^{[1]}$, our data revealed a significant increase in blood lactate levels and impairment in $\mathrm{SCVO}_{2}$ during the IPO period in comparison with preoperative values. According to Ranucci et al. ${ }^{[13]}, \mathrm{ScvO}_{2}$ and blood lactate level used in combination could be a clinical tool to help discern if an elevated lactate level is due to hypoperfusion or other mechanisms. In our study, a strong negative correlation supported the relationship between a $\mathrm{ScvO}_{2}$ drop below $65 \%$ 
Table 3. Postoperative clinical variables

\begin{tabular}{l|c|c}
\hline Variables & $\begin{array}{c}\text { S-NPPV Group } \\
(\mathbf{n = 2 0 )}\end{array}$ & $\begin{array}{c}\text { E-NPPV Group } \\
(\mathbf{n = 2 1 )}\end{array}$ \\
\hline Atelectasis \% (n) & $26.3(5)$ & $4.7(1)^{*}$ \\
\hline Pleural effusion \% (n) & $15.7(3)$ & $9.5(2)$ \\
\hline Pneumonia \% (n) & $15.7(3)$ & $4.7(1)^{*}$ \\
\hline Mechanical ventilation time (h) & $12.1 \pm 1.8$ & $11.2 \pm 1.1$ \\
\hline Postoperative hospital stay (days) & $8.1 \pm 2.1$ & $6.9 \pm 1.3^{*}$ \\
\hline
\end{tabular}

Data are shown as mean \pm standard deviation. Comparison between the groups

${ }^{*} P<0.05$. E-NPPV=extended-time noninvasive positive pressure ventilation; S-NPPV=short-time noninvasive positive pressure ventilation

and an increase in blood lactate levels. For this reason, we assume that blood lactate levels found in the current study were associated with impairment in tissue perfusion.

After NPPV application, there were a significant drop in blood lactate levels and a significant improvement in $\mathrm{ScvO}_{2}$ values in the E-NPPV group when compared with the S-NPPV group. Based on these findings, we believe that NPPV was the interventional mechanism for improvement in tissue perfusion in patients undergoing CABG, in particular E-NPPV.

In general, some potential mechanisms may explain our findings. Firstly, we believe that NPPV promoted a beneficial effect on cardiac function. After on-pump CABG, early reperfusion precipitates a period of relative hemodynamic instability in which small and rapid changes in ventricular loading, myocardial perfusion pressure, and endogenous inotropic state can change global ventricular performance considerably ${ }^{[14]}$. In addition, the early reperfusion period is characterized by a high incidence of regional or global ventricular dysfunction. Even in patients with a preoperative preserved ventricular function, a significant ejection fraction reduction could be noted in the first four hours after surgery ${ }^{[15]}$. Previous research has shown that NPPV application in patients with cardiac dysfunction could increase cardiac index systemic oxygen delivery, and oxygen consumption ${ }^{[16-19]}$. Despite the fact that patients in the present study had preserved cardiac function, we believe that NPPV could have prevented acute cardiac alterations related to the operation. Secondly, studies have shown experimentally that atelectasis causes a significant increase in right ventricular afterload, thereby affecting left ventricular performance. This effect of atelectasis on right ventricular afterload during mechanical ventilation could be explained by two mechanisms: overdistention in aerated lung areas and local hypoxic pulmonary vasoconstriction in nonaerated lung areas ${ }^{[20,21]}$. In the current study, the E-NPPV group demonstrated a lower prevalence of atelectasis than the S-NPPV group. We speculate that E-NPPV reduced the risk of atelectasis and subsequently right ventricular stress by a reduction in hypoxic pulmonary vasoconstriction.

Zarbock et al. ${ }^{[11]}$ demonstrated that prophylactic NPPV application at least six hours after the operation increased pulmonary oxygen transfer, reduced pulmonary complications, and also decreased ICU readmission rates following elective cardiac surgery. Similar results were found in our study. The use of E-NPPV was associated with a significantly better preservation of pulmonary function than the S-NPPV.

A greater degree of pulmonary dysfunction increases the risk of pulmonary complications during the CABG postoperative course, which may result in longer postoperative hospital stay and increased mortality ${ }^{[4-6]}$. These results are in agreement with our findings; the S-NPPV group presented with a higher pulmonary function impairment which was associated with a significantly greater intubation time, occurrence of atelectasis, and pneumonia until POD5, and longer hospital stay than the E-NPPV group.

We were able to show that E-NPPV application significantly preserved pulmonary function and reduced respiratory events in patients deemed to be at low surgical risk. The literature had demonstrated that high-risk patients could potentially have more benefits with NPPV application ${ }^{[7]}$. Therefore, we speculate that the benefit of E-NPPV in high-risk patients following cardiac surgery could be even more pronounced, which may also lead to a more profound reduction in length of hospital stay.

\section{Limitations}

Our study has some limitations that should be highlighted. The duration (one hour, twice a day) of NPPV after ICU discharge in the E-NPPV group may not have been enough to promote a faster return of the pulmonary function to baseline values. However, the E-NPPV protocol was able to promote a better preservation of pulmonary function and to prevent postoperative complications when compared to the S-NPPV.

\section{CONCLUSION}

Prophylactic E-NPPV administered in the early postoperative period of CABG resulted in greater improvements in tissue perfusion, pulmonary function, and clinical outcomes than S-NPPV in patients with preserved left ventricular function. These findings hold clinical relevance and should be considered when developing the care plan for individuals undergoing a major cardiac surgery. 


\section{Authors' roles \& responsibilities}

MLSN Concept, design, acquisition, analysis and interpretation of data, critical review of the study; final approval of the manuscript version to be published

DWB Interpretation of data, and critical review of the study; final approval of the manuscript version to be published

YGL Acquisition of data, final approval of the manuscript version to be published

FSP Acquisition of data, final approval of the manuscript version to be published

RA Critical review of the study, final approval of the manuscript version to be published

PRLL Acquisition of data, final approval of the manuscript version to be published

GF Acquisition of data, final approval of the manuscript version to be published

AMCS Analysis and interpretation of data; final approval of the manuscript version to be published

NOM Interpretation of data, critical review of the study; final approval of the manuscript version to be published

$\mathrm{NH}$ Critical review of the study, final approval of the manuscript version to be published

SG Concept, design, analysis and interpretation of data, critical review of the study; final approval of the manuscript version to be published

WJG Concept, design, analysis and interpretation of data, critical review of the study; final approval of the manuscript version to be published

\section{REFERENCES}

1. Holm J, Hakanson E, Vánky F, Svedjeholm R. Mixed venous oxygen saturation predicts short- and long-term outcome after coronary artery bypass grafting surgery: a retrospective cohort analysis. $\mathrm{Br} J$ Anaesth. 2011;107(3):344-50.

2. Chiumello D, Chevallard G, Gregoretti C. Non-invasive ventilation in postoperative patients: a systematic review. Intensive Care Med. 2011;37(6):918-29.

3. Hu BY, Laine GA, Wang S, Solis RT. Combined central venous oxygen saturation and lactate as markers of occult hypoperfusion and outcome following cardiac surgery. J Cardiothorac Vasc Anesth. 2012;26(1):52-7.

4. Guizilini S, Alves DF, Bolzan DW, Cancio AS, Regenga MM, Moreira RS, et al. Sub-xyphoid pleural drain as a determinant of functional capacity and clinical results after off-pump coronary artery bypass surgery: a randomized clinical trial. Interact Cardiovasc Thorac Surg. 2014;19(3):382-7.

5. Guizilini S, Bolzan DW, Faresin SM, Ferraz RF, Tavolaro K, Cancio AA, et al. Pleurotomy with subxyphoid pleural drain affords similar effects to pleural integrity in pulmonary function after off-pump coronary artery bypass graft. J Cardiothorac Surg. 2012;7:11.

6. Guizilini S, Gomes WJ, Faresin SM, Bolzan DW, Buffolo E, Carvalho AC, et al. Influence of pleurotomy on pulmonary function after off-pump coronary artery bypass grafting. Ann Thorac Surg. 2007;84(3):817-22.

7. Chen XF, Ye JL. Efficacy and safety of non-invasive positive pressure ventilation in the care of dyspnea after cardiac surgery. Zhongguo Wei Zhong Bing Ji Jiu Yi Xue. 2007;19(9):542-5.

8. Standardization of Spirometry, 1994 Update. American Thoracic Society. Am J Respir Crit Care Med. 1995;152(3):1107-36.

9. MurphyTF, Sethi S. Bacterial infection in chronic obstructive pulmonary disease. Am Rev Respir Dis. 1992;146(4):1067-83.

10. Vargas FS, Terra-Filho M, Hueb W, Teixeira LR, Cukier A, Light RW. Pulmonary function after coronary artery bypass surgery. Respir Med. 1997;91 (10):62933.

11. Zarbock A, Mueller E, Netzer S, Gabriel A, Feindt P, Kindgen-Milles D. Prophylactic nasal continuous positive airway pressure following cardiac surgery protects from postoperative pulmonary complications: a prospective, randomized, controlled trial in 500 patients. Chest. 2009:135(5):1252-9.

12. Pölönen P, Ruokonen $E$, Hippeläinen M, Pöyhönen M, Takala J. A prospective, randomized study of goal-oriented hemodynamic therapy in cardiac surgical patients. Anesth Analg. 2000;90(5):1052-9.

13. Ranucci M, Isgrò G, Carlucci C, De La Torre T, Enginoli S, Frigiola A; Surgical and Clinical Outcome REsearch Group. Central venous oxygen saturation and blood lactate levels during cardiopulmonary bypass are associated with outcome after pediatric cardiac surgery. Crit Care. 2010;14(4):R149.

14. Leung JM, O'Kelly B, Browner WS, Tubau J, Hollenberg M, Mangano DT. Prognostic importance of postbypass regional wall-motion abnormalities in patients undergoing coronary artery bypass graft surgery. SPI Research Group. Anesthesiology. 1989;71(1):16-25.

15. Mangano DT. Biventricular function after myocardial revascularization in humans: deterioration and recovery patterns during the first 24 hours. Anesthesiology. 1985;62(5):571-7.

16. Kallet RH, Diaz JV. The physiologic effects of noninvasive ventilation. Respir Care. 2009;54(1):102-15.

17. Acosta MF, DiBenedetto R, Rahimi A, Acosta MF, Cuadra O, Van Nguyen A, et al. Hemodynamic effects of noninvasive bilevel positive airway pressure on patients with chronic congestive heart failure with systolic dysfunction. Chest. 2000;118(4):1004-9.

18. Baratz DM, Westbrook PR, Shah PK, Mohsenifar Z. Effect of nasal continuous positive airway pressure on cardiac output and oxygen delivery in patients with congestive heart failure. Chest.1992;102(5):1397-401.

19. Buda AJ, Pinsky MR, Ingels NB Jr, Daughters GT 2nd, Stinson EB, Alderman EL. Effect of intrathoracic pressure on left ventricular performance. N Engl J Med. 1979;301(9):453-9.

20. Duggan M, McCaul CL, McNamara PJ, Engelberts D, Ackerley C, Kavanagh BP. Atelectasis causes vascular leak and lethal right ventricular failure in uninjured rat lungs. Am J Respir Crit Care Med. 2003;167(12):1633-40.

21. Creamer KM, McCloud LL, Fisher LE, Ehrhart IC. Ventilation above closing volume reduces pulmonary vascular resistance hysteresis. Am J Respir Crit Care Med.1998;158(4):1114-9. 